\title{
A New Product Development and Effect Analysis of Tinnitus Therapy Based on Pink Noise Tone
}

Hongbo Lai

University of Shanghai for Science and Technology

Gaoxing Wang

University of Shanghai for Science and Technology

Meijuan Gao

University of Shanghai for Science and Technology

Yang Zhou ( $\sim$ zhouyang@usst.edu.cn )

Business School, University of Shanghai for Science and Technology, Shanghai, China

Shuai Wu

Zhongshan Hospital, Fudan University

Jianling Wang

Shanghai Open University

\section{Research Article}

Keywords: Pink Noise, Tinnitus, Music Therapy, Therapeutic Effect

Posted Date: January 21st, 2021

DOI: https://doi.org/10.21203/rs.3.rs-146522/v1

License: (c) (i) This work is licensed under a Creative Commons Attribution 4.0 International License.

Read Full License 


\section{Abstract}

Sound therapy for tinnitus includes not only acclimatization therapy and masking therapy, but also music therapy. Traditional music therapy for tinnitus is mostly based on "white noise". This study attempts to introduce pink noise into the field of tinnitus rehabilitation therapy. The semi-duplicate tone produced on the basis of fractal technology (fractal mathematics) is used as the basic tone of tinnitus rehabilitation treatment. It is found that pink noise has better inhibition function for tinnitus. At the same time, 30 adult patients (17 males and 13 females) with tinnitus and hearing loss of varying degrees were selected as the research subjects. The clinical efficacy of tinnitus symptoms was evaluated by fractal music program of hearing aids. The test results showed that there was a significant correlation between the score before admission and the change of $P$ value after treatment. At the same time, further analysis shows that white noise (left) and pink noise (right) are the spectrograms of basic tunes. The frequency component power of pink noise mainly distributes in the middle and low frequency bands. It is the slope of $-3 \mathrm{~dB} /$ Oct. It attenuates downward with its multiple frequency. That is to say, pink noise hears a flat frequency response in the human ear, that is, "a very pleasant noise". In this study, for tinnitus treatment of this "pink noise" tune, there are two forms of implementation, one is the hardware integrated circuit chip, that is, similar to portable hearing aids equipment, for professional physicians to examine. Secondly, APP based on Android and IOS system produces fractal melody simulating pink noise by using semi-replication algorithm (fractal mathematics principle), which provides patients with mild tinnitus symptoms and no need of long-term treatment, and finally eliminates the discomfort caused by tinnitus.

\section{Objective}

Music therapy, as well as retraining therapy and masking therapy, is the common method that modern otologists used to treat tinnitus patients. Most of the traditional music therapies for tinnitus use "white noise" as the basic tone. Nevertheless, the efforts to explore other kinds of noise that has a better curative effect on tinnitus is still in need. This paper aims to apply "pink noise" into the tinnitus rehabilitation therapy field to explore whether the tone has a better inhibitory effect on tinnitus.

\section{Methods}

We produce the semi-duplicate tone based on fractal technology (fractal mathematics and use this semiduplicate tone as the basic tone.34 adult tinnitus patients with vary degrees of hearing loss are selected as the research subjects. The patients are treated with music therapy by the fractal music program of hearing aids, and adopt the evaluation of the curative effect for their tinnitus 1 month, 3 months and 6 months after the treatment respectively.

\section{Results}

The result shows that there is a significant difference between the score before the treatment and that after the treatment $(P<0.05)$. In addition, by further comparing the spectrograms of tones based on white noise and pink noise, it finds that the frequency component power of pink noise mainly distributes in the 
middle and low frequency bands. Its slope is $-3 \mathrm{~dB} / 0 \mathrm{ct}$, which means the frequency decay downward with this multiple. That is to say, pink noise was heard as a flat frequency response in the human ear, that is, "a very pleasant noise".

\section{Conclusions}

Finally, this paper suggests two kinds of new tinnitus treatment products based on the "pink noise", one is the hardware integrated circuit chip, that is, similar to portable hearing aids equipment, targeting at the diagnosis process of professional aurist otologists; the other one is, the APP based on Android and IOS system, targeting at the patients with mild tinnitus symptoms and no need of long-term treatment, helping them eliminate the discomfort caused by tinnitus.

\section{Introduction}

Tinnitus is a kind of sound that patients feel when there is no external sound source. It is reported that the global incidence of adult tinnitus is $10 \%$ to $15 \%$, and $20 \%$ of these patients need clinical intervention [1]. The incidence of tinnitus in people over 60 years old is reported to be $14.5 \%$ in China. There are three reasons for the increase in tinnitus: the first is the deepening of social aging degree; the second is the impact of urban pollution, especially noise pollution; and the third is the extensive use of all kinds of vehicles. Accordingly, the tinnitus population who need intervention treatment increases.

Tinnitus itself is not a disease, but a symptom. It is the sound perception of the ear or head when these is no external sound. Its occurrence is the result of many factors, and sometimes it is the precursor of a disease. The incidence of tinnitus has gradually increased in recent years [2]. Studies have shown that tinnitus not only affects patients' language communication and sleep quality, but also causes anxiety, depression and even suicidal tendencies in patients with chronic tinnitus $[3,4]$. Tinnitus is one of the three most common complications in otology. The number of tinnitus patients is quite large, and the prevalence rate is usually $10 \%-15 \%[5]$.

In the course of this new product trial, a global COVID-19 outbreak. Going to the hospital for a follow-up is a greater risk of infection. In this case, the doctor is difficult to grasp the patient's condition, and timely adjustment of treatment program. Based on this, in the course of this experiment, we contact the relevant departments of the hospital to take measures to ensure that doctors can timely access to patients with tinnitus. To channel the patients' emotions in a proper way and reduce the negative impact of the epidemic on their emotions. So that all participants can maintain an optimistic attitude to receive treatment, to maximize the efficacy of the product.

\section{Current common methods and analysis of tinnitus treatment}

\section{Causes and treatment of tinnitus}

With the development of society and the acceleration of life rhythm, the incidence of tinnitus is increasing year by year. It seriously affects the people's body, mind and life. According to survey study of Ma and 
Zhu (2009), 2.4\% of the tinnitus patients would seek for hospital treatment [6]. Therefore, it is a pressing problem for the medical community to explore effective methods to prevent and treat tinnitus. Tinnitus is only a sensation, rather than sound hallucinations or illusions. At present, there is no evidence of visual perception about tinnitus in medicine. There are different opinions on tinnitus's pathogenic site and its lesion physiological changes. Experts generally believe that tinnitus is not an independent disease [7]. Tinnitus can be caused by the lesions in any part of the auditory system, meanwhile, it sometimes can also be a concomitant symptom of systemic diseases. The etiology and influencing factors of tinnitus are diverse, so it is difficult to find its exact cause [8].

The pathogenesis of tinnitus is complex and related to many diseases[9]. Moreover, due to the unclear mechanism, there is still a lack of specific treatment methods and drugs at this stage. As a result, the treatment process of tinnitus is also diversified. From the perspective of medicine, tinnitus treatment commonly focuses on the physiological treatment, such as surgical removal of acoustic nerve tumors, including drug therapy [10] and surgery, so as to achieve the purpose of treating tinnitus. But in the field of audiology, another more commonly used method to treat tinnitus called "tinnitus management", which focuses on the improvement of the overall condition, i.e. physiological and psychological condition after the treatment[11], including counseling, cognitive behavioral therapy[12], acclimation therapy, application of hearing aids, tinnitus masks and auxiliary devices, etc. In conclusion, there are two principles for the treatment of tinnitus at this stage. The first is etiological treatment, which means to treat the protopathy of the tinnitus. After the treatment of the protopathy, there still will be long-term severe tinnitus. The cause of this long-term tinnitus is unclear, but it will remain exist seriously for quite a long time. The second is symptomatic treatment, including tinnitus retraining therapy, masking therapy, music therapy, relaxation therapy and so on.

\section{Sound-based therapy: tinnitus retraining therapy and masking therapy}

There are two recognized and widely used methods in sound therapy of tinnitus, namely tinnitus retraining therapy and tinnitus masking therapy. The former is widely used in the world, while the latter is commonly used in some areas, such as China [13]. Tinnitus retraining therapy (TRT) is a therapy based on the tinnitus neurophysiological model. Its purpose is not to eliminate the production of sound, but to block the tinnitus-related sound signals (the activity of tinnitus-related neuron) transmitted from the auditory pathway to other brain systems, especially the limbic system and autonomic nervous system. Xu and $\mathrm{Bu}$ (2012) stated that tinnitus retraining therapy belongs to the incomplete masking therapy and proposed that masking the sound intensity cannot actually cover up tinnitus but coexist with tinnitus[14]. Through long-term retraining training (psychological adjustment, diversion of attention and sound therapy, etc.), the central sensitivity of the patients would reduce, which makes the patients adapt to the tinnitus. The method has been widely used in the treatment of tinnitus [15]. Jastreboff PJ (2013) believes that TRT is suitable for any type of tinnitus treatment [16].

The principle of tinnitus masking therapy is, making the tinnitus patient always in a circumstance of some small volume natural voice, rather than an absolutely quiet circumstance, by which the patient's 
attention could divert from the tinnitus and the patient's tension could relax. This therapy can also improve insomnia, depression, anxiety and other non-auditory problems in patients with severe tinnitus [17]. At present, masking therapy is widely used in clinical treatment of tinnitus patients in China. Masking therapy is also a physiological treatment. It is simple, safe, and with fewer adverse reactions. However, masking therapy can only work on a particular kind of tinnitus patients. Moreover, if the patients leave the masking, tinnitus will still occur. As thus, masking therapy cannot achieve the desired therapeutic effect.

There are three goals of using sound to treat tinnitus, no matter with tinnitus retraining therapy or masking therapy. Firstly, to reduce stress and tension caused by tinnitus. Secondly, to divert patients' attention from tinnitus by reducing the difference between tinnitus and ambient sound. Thirdly, to actively distract patient from the tinnitus. This study investigated the tinnitus rehabilitation technology used by different hearing aid manufacturers. Given Sonova in Switzerland and Audikang in Denmark as examples, the hearing aids of these companies adopted the traditional "masking" technology. The devices in fact cover up the tinnitus with a louder voice, in order to help the patients ignore the existence of tinnitus. This also exposed the limitations of masking technology. After a period of time, tinnitus often occurs again and produces a new round of tinnitus. At this time, patients often feel an even louder tinnitus than before (i.e. a "rebound"). Consequently, the patients need a higher decibel of sound to mask tinnitus, which can lead to repeated tinnitus symptoms, and even cause hear loss or other secondary damages to patients.

\section{Emerging Application of music therapy in tinnitus treatment: From white noise to pink noise}

\section{Application of music therapy in tinnitus treatment}

The methods of sound therapy for tinnitus includes not only tinnitus retraining therapy and masking therapy, but also music therapy. In recent years, with the change of medical model, people's psychological and social attributes have attracted more and more attention. Therefore, the great importance is gradually given to the music therapy. This therapy is considered to be creative, flexible, safe and effective. Music has a relaxing effect, so that patients can temporarily ignore the existence of tinnitus. This music therapy has been widely used in the world and its effect has been verified. Studies have shown that the brain dominates music activities, and many human emotions and behaviors are under the control of the right hemisphere [18]. Music plays a direct role on the right hemisphere of the brain, which can regulate human emotions and behaviors. This is an important basis for the principles of music therapy. Music therapy is a kind of therapy which takes music activities as the medium and influences the human body through the characteristics of music to help patients achieve the integration of physiology, psychology and emotion in the course of treatment, thus eliminating psychological obstacles and restoring or improving mental and physical health [19]. Zhou and Ma (2012) found that the relaxation training of music is very helpful to adjust the mentality of tinnitus patients [20].

Christoph et al. (2015) studied on the neurological basis of Heidelberg music model in the treatment of tinnitus[21]. Fifty patients with acute tinnitus were randomly divided into treatment group and control 
group. The treatment group was treated with Heidelberg music model. Before and after treatment, MRI and noise levels were measured and analyzed. The results showed that the structural gray matter in the precuneus, medial frontal lobe and auditory cortex of the treatment group was higher than that of the control group.

\section{The limitations of current music therapy and the "pink noise"}

Simple music therapy for tinnitus has been discussed for a long time in clinical research. The sound of beautiful external music can attract the patients' attention from the tinnitus and reduce patients' perception of tinnitus. In this way, patients would temporarily ignore the tinnitus and gradually adapt to tinnitus. Recently, music therapy is playing an important role in tinnitus treatment. However, previous research also showed that if the patients listen to the same piece of music for a long term, their sensitivity of hearing will reduce, due to auditory fatigue. Furthermore, because the background noise of hearing equipment is uncontrolled, the patients are easy to get further hearing loss. Therefore, it is called for more efforts to spend on exploring other types of music that can also be used as the base note in the tinnitus treatment, with the similar or even better curative effect.

At present, in the treatment of tinnitus, most of the tones are based on "white noise". White noise refers to the sound in which the power of the frequency component is uniform in the whole audible range. As the human ear is sensitive to high frequency, this "white noise" sounds very noisy rustle. The white noise is recognized as an effective "sound cosmetic treatment" for nervous system diseases such as mental distraction, tinnitus, auditory hypersensitivity and hyperactivity disorder.

Referring to the latest findings in audiological research, we find that the frequency component power of pink noise mainly distributes in the middle and low frequency bands, which have better suppression function for tinnitus. Based on this, the semi-duplicated "pink noise" was used as the basic tone in this study, aiming to optimize the performance of tinnitus rehabilitation. On the other hand, from the perspective of waveform, pink noise is fractal. The audio data within a certain range has the same or similar energy. Fractal music is more effective than white noise in the treatment of tinnitus, which is also recognized by international audiologists. Meanwhile, pink noise can be used to simulate natural sounds such as waterfalls or rain. This kind of sound can soothe people's emotions and make people relax.

\section{Data And Methods}

\section{Data collection and experiment}

The scheme of this experiment was approved by the Ethics Committee of Zhongshan Hospital Affiliated to Fudan University. All processes are carried out in strict accordance with relevant standards and regulations. All participants were adults over 18 years old. And before the experiment, we have obtained the informed consent of all the participants. 34 adult patients with tinnitus and hearing loss were selected as the research objects. They were all patients with subjective tinnitus who had serious tinnitus related disorders and were willing to cooperate with this study. All the participants completed the tinnitus 
disability assessment scale to assess their actual hearing loss, and then wear hearing aids to compensate for hearing loss. The hearing aid is based on "pink noise", and fractal music is formed by fractal algorithm. The fractal music program is used for sound therapy. The clinical efficacy of tinnitus symptoms is evaluated at 1, 3 and 6 months after treatment. In addition, the experimental subjects of this product do not include objective tinnitus patients and tinnitus patients who participate in similar studies at the same time.

\section{Statistical treatment}

We collected the THI score of each sample patient, 1 month, 3 months and 6 months after the treatment respectively, and calculate the mean (M) and standard deviation (SD), the details are shown in Table 1. Table 2 shows the results of paired sample t-test on THI scores before and after music therapy for 1,3 and 6 months by spss23.0. The results show that there was a significant correlation between $P$ values of the scores before and after the treatment.

\section{Table 1 Mean (M) and standard deviation (SD) of tinnitus disability assessment score before and after treatment}

\begin{tabular}{|llllllll|}
\hline \multicolumn{2}{|c|}{ Score before treatment } & THI1 & \multicolumn{3}{c|}{ THI2 } & \multicolumn{3}{c|}{ THI3 } \\
\hline M & SD & M & SD & M & SD & M & SD \\
\hline 65.6 & 19.0 & 38.8 & 16.9 & 22.7 & 10.4 & 42.0 & 15.5 \\
\hline
\end{tabular}

Table 2 Results of pared-samples T test: 1 month, 3 months and 6 months after the treatment

\begin{tabular}{|lll|}
\hline & F & P \\
\hline THI 1 & 4.808 & 0.000 \\
THI 2 & 9.328 & 0.000 \\
THI 3 & 6.888 & 0.000 \\
\hline
\end{tabular}

The spectrums of voice that used white noise (left) and pink noise (right) as the basic tunes are shown in Figure 1. From the spectrum of the two kinds of noise, the power of a sound frequency component of white noise is uniform in the whole audible range $(20 \mathrm{~Hz}-20 \mathrm{kHz})$. As the human ear is more sensitive to high frequency, this kind of sound sounds very noisy rustle. Pink noise, on the other hand, attenuates downward at multiple frequencies with the slope of $-3 \mathrm{~dB} /$ Oct. The frequency component power of pink 
noise mainly distributes in the middle and low frequency bands. Pink noise hears a flat frequency response in the human ear, which seems to be "a very pleasant noise".

Further, we found that when tinnitus patients with normal hearing were examined, it was found that the amplitude of high-frequency region $(1 \mathrm{kHz}-4 \mathrm{kHz})$ decreased significantly, and the slope of DPOAE function curve of corresponding frequency increased. In other words, the trend of the energy and power of pink noise and trend of tinnitus amplitude are similar to each other. Therefore, pink noise is less harmful to the tinnitus patients' hearing than white noise.

\section{Therapeutic program procedure}

We proposed corresponding targeted treatment for the tinnitus patients with different tinnitus level. The targeted treatment is based on both tinnitus retraining therapy (TRT) and cognitive behavioral therapy (CBT). The treatment procedure is as follows: (1) Hearing assessment and tinnitus assessment (THI survey assessment); (2) Judging tinnitus level; (3) Developing appropriate treatment plans; (4) Counseling (tinnitus mechanism, relationship between tinnitus and deafness, factors affecting tinnitus and answering users' questions); (5) Adjusting (adjusting the volume of a hearing aid according to patient's hearing); (6) Stress management (guiding relaxation exercises according to the users' situations); (7) Following (interviewing each sample patient and evaluating their THI 1, 3, 6 months respectively after the treatment; giving guidance for next step based on the final results). The details are shown in Figure 2.

The "pink noise" tune for tinnitus therapy can be produced by two ways in the R\&D process. The first is integrated circuit chips hardware, a device similar to hearing aids. This hardware is provided to patients who need tinnitus interventions after examination by professional doctors. The second is the APP based on Android and IOS system that the patients can download and use. It is an APP that uses the semireplication algorithm (fractal mathematics principle) to produce the fractal music of the analog pink noise. On the one hand, the integrated circuits generate fractal music with pink noise as keynote by the semi-duplicate complex chip technology based on fractal computing technology. And then it combines tinnitus retraining therapy (TRT) and cognitive behavioral therapy (CBT) to improve and innovate tinnitus retraining therapy technology. On the other hand, the APP is provided to the patients with mild tinnitus symptoms but no need for long-term treatment. After judging tinnitus level, the APP could be recommended by volunteers to the patients and the patients could downloaded it from the APP Store. In addition, APP can help people who are inconvenient to go out but have sudden tinnitus in special periods such as epidemic situation. Let these people understand the relevant knowledge of tinnitus, and update some methods of relieving tinnitus at home from time to time.

\section{Discussion}

Seen from the development history of tinnitus treatment, music therapy is a promising one that may become an important method for the tinnitus treatment in the future. Many previous studies have shown that music therapy can change the activity of brain limbic system by increasing peripheral sound signal 
input. Thus, the subjective tinnitus of patients can be greatly relieved [22]. Of course, further neurophysiological research and a large number of clinical observations are needed before it becomes the routine clinical diagnosis and treatment of tinnitus. The tinnitus rehabilitation products proposed in this study are based on semi-duplicated tunes. It uses pink noise as the keynote, by which can optimize the tinnitus rehabilitation performance, so as to increase the product variety of the tinnitus rehabilitation products in the future market.

Discussions (1) Pink noise has been widely used in audiometry and ear diseases examination. On this basis, this study introduced pink noise into the field of tinnitus rehabilitation therapy. The semi-duplicated tones generated on the basis of fractal technology (fractal mathematics) are used as the basic sound of tinnitus rehabilitation therapy. During the experiment, the patients are given some subtle and soft tones depending on their personal hearing conditions. This subtle and soft tones could stimulate the brain, by which the activity of neurons would be enhanced and the sensitivity of the brain will be reduced. As the brain will eventually become accustomed to any predictable sound, the impact of stimulation on emotional state may be reduced. No matter how painful tinnitus is, the method can always be used.

Discussions (2) This study developed two tinnitus rehabilitation products with pink noise as the keynote. The pressure on music from this "pink noise" is predictable, but not repeatable, so as to achieve a good expected therapeutic effect. At the same time, this voice program is designed based on fractal technology, the rhythm and sound intensity of which can be adjusted by the personalized needs of tinnitus patients. The tinnitus rehabilitation products provide similar music sounds, using semi-duplicate generation method. The stimulating tones are similar, but not identical. It could reduce the risk of tone boredom, and also could personalize to meet the needs and preferences of patients.

Discussions (3) The products of this study will be wearable for patients. The patients can use them at anytime and anywhere. The equipment can also be shared by several people (not at the same time). In the use process, first help patients to understand the tinnitus type, frequency, loudness, and then use the targeted products. At the same time, the tinnitus APP software tool is further developed by collecting the relevant data of product use. The APP can improve the autonomic nervous system response induced by tinnitus. Such as changing the concentration of some neurotransmitters to reduce tension and anxiety and emergency response [23], and become a daily training and help practice decompression tools. Finally, tinnitus management is realized step by step to eliminate the discomfort caused by tinnitus. During the new crown epidemic, some patients had good feedback.

Discussion (4) In the process of this study, the global epidemic broke out. Combined with the actual situation to show the corporate social responsibility, we take a series of measures, including but not limited to APP function expansion. On the one hand, it provides "non-contact" communication channels between patients and doctors. On the other hand, updated from time to time some practical home suggestions to help those people with tinnitus caused by the epidemic, has been unanimously praised by the public. 
In conclusion, this paper develops a method and service for tinnitus treatment based on the music therapy with pink noise as keynote, which mainly solves the embarrassment of no specific drugs and no special equipment for tinnitus treatment. At the same time, it contributes to speed up the development of medical devices, greatly reduce the using cost, make the devices more affordable, and improve the quality of tinnitus patients' life. At the same time, tinnitus treatment is also a long-term work, which cannot be overcome in the short term. As more and more patients no longer conflict and pay attention to tinnitus, especially more and more people use products. We can get more data and carry out targeted product iterative innovation, including the optimization of new algorithms. In the future, we will further improve our products and services.

\section{Declarations}

\section{Author Contributions}

Conceptualization: Hangbo Lai, Meijuan Gao, Yang Zhou, Shuai Wu

Data curation: Gaoxing Wang, Meijuan Gao, Jianling Wang

Formal analysis: Hangbo Lai, Meijuan Gao, Gaoxing Wang

Funding acquisition: Hangbo Lai

Investigation: Meijuan Gao, Hangbo Lai, Gaoxing Wang, Jianling Wang

Methodology: Hangbo Lai, Meijuan Gao, Jianling Wang

Project administration: Hangbo Lai, Yang Zhou

Resources: Meijuan Gao

Supervision: Hangbo Lai, Yang Zhou

Validation: Hangbo Lai, Meijuan Gao

Writing-original draft preparation: Hangbo Lai, Meijuan Gao, Gaoxing Wang

Writing-review \& editing: Yang Zhou, Hangbo Lai

\section{Disclosure}

The authors report no conflicts of interest in this work.

\section{Funding}

This report was supported by a grant (2020JG016-BJB560) from the General project fund of philosophy and social sciences of Shanghai in 2020. 


\section{References}

1. Lenarz T. Guidelines for the diagnosis and treatment of tinnitus (German society for otolaryngology, head and neck surgery). Deutsche Medizin. 2000;17: 14-18.

2. Yu J, Sun Y, Zhang Y, Zhang S, He Y, et al. Clinical investigation on effects of lipo-prostagland in E1 combined with hyperbaric oxygen therapy in 96 patients with sudden deafness and tinnitus. Clinical Misdiagnosis \& Mistherapy. 2020;23: 314-316.

3. Li Y, Tang Z, Yu X. Survey and clinical feature analysis of the aged subjective tinnitus in a community. Journal of Southern Medical University. 2013;33: 1243-1245.

4. Nondahl DM, Cruickshanks KJ, Huang G, Klein BE, Klein R, et al. Tinnitus and its Risk Factors in the Beaver Dam Offspring Study. International Journal of Audiology. 2011;50: 313-320.

5. Tunkel DE, Bauer CA, Sun GH, Rosenfeld RM, Chandrasekhar SS, et al. Clinical practice guideline: Tinnitus. Otolaryngology- Head and Neck Surgery. 2014;151: 1-40.

6. Ma J, Zhu X. Progression of investigation in the treatment of tinnitus with TCM. Chinese Archives of Traditional Chinese Medicine. 2009;27: 588-590.

7. Gong S, Yu Q. Current status of diagnosis and treatment of tinnitus. Chinese Journal of Otorhinolaryngology Head Neck Surgery. 2012;47: 788-792.

8. Li M, Huang J. Re-recognition of diagnosis and treatment of tinnitus. Chinese Journal of Otorhinolaryngology Head Neck Surgery. 2009;44: 701-704.

9. Zhong C. Use MP3 instead of tinnitus mask to treat tinnitus. Chinese Journal of OtorhinolaryngologySkull Base Surgery. 2005;11: 443.

10. Tyler RS. Patient preferences and willingness to pay for tinnitus treatments. Journal of the American Academy of Audiology. 2012;23: 115-125.

11. TUNKEL D E, BAUER C A, SUN G H, et al. Clinical practice guideline: tinnitus. Otolaryngology- Head Neck Surgery. 2014;151 (2Suppl: S1-S40).

12. Jiang D. The differences between tinnitus and decreased sound tolerance: Understanding and management. Chinese Scientific Journal of Hearing and Speech Rehabilitation. 2012;10: 375-378.

13. Lu Y. Clinical treatment observation of chronic subjective tinnitus treated by masking therapy and tinnitus retraining therapy. Journal of Clinical and Experimental Medicine. 2016;15: 2275-2277.

14. Xu X, BU X. Progress in sound therapy for tinnitus. Chinese Journal of Otolaryngology Integrated Medicine. 2012;20: 388-390.

15. Bauer C, Brozoski T. Effect of tinnitus retraining therapy on the loudness and annoyance of tinnitus: a controlled trial. Ear Hear. 2011;32: 145-155.

16. Jastreboff P, Jastreboff M. Using TRT to treat hyper-acusis, misophonia and phonophobia. ENT and Audiology News. 2013;880.

17. Mazurek B, Fischer F, Haupt H, Georgiewa P, Reisshauer A, et al. Amodified version of tinnitus retraining therapy: Observing long-term outcome and predictors. Audio Neurotol. 2006;11(5): 276- 
286.

18. Yang X, Wang J, Lai W. The Application and development of music therapy in the field of pain. International Journal of Stomatology. 2013;40: 513-515.

19. Zhang L, Zhang M, Zhang B. Analysis of the mechanism of music therapy and its application in the treatment of insomnia. Liaoning Journal of Traditional Chinese Medicine. 2010;37: 420-422.

20. Zhou J, Ma Y. Tinnitus retraining therapy: a clinical control study of 52 cases. Chinese Journal of Otorhinolaryngology $\otimes$ Skull Base Surgery. 2012;18: 198-200.

21. Krick CM, Grapp M, Daneshvar-Talebi J, Reith W, Plinkert PK, et al. (2015) Cortical reorganization in recent-onset tinnius patients by the Heidelberg Model of Music Therapy. 2015;9: 41-49.

22. Kuk F, Peeters H, Lau CL. The efficacy of fractal music employed in hearing aids for tinnitus management. Hearing Review. 2010;17(10):32 42.

23. Sweetow RW, Kuk F, Caprioli S. A controlled study on the effectiveness of fractal tones and Widex Zen Therapy on subjects with minimal need for amplification. Hearing Review. 2015;22(9): 30 36.

\section{Figures}
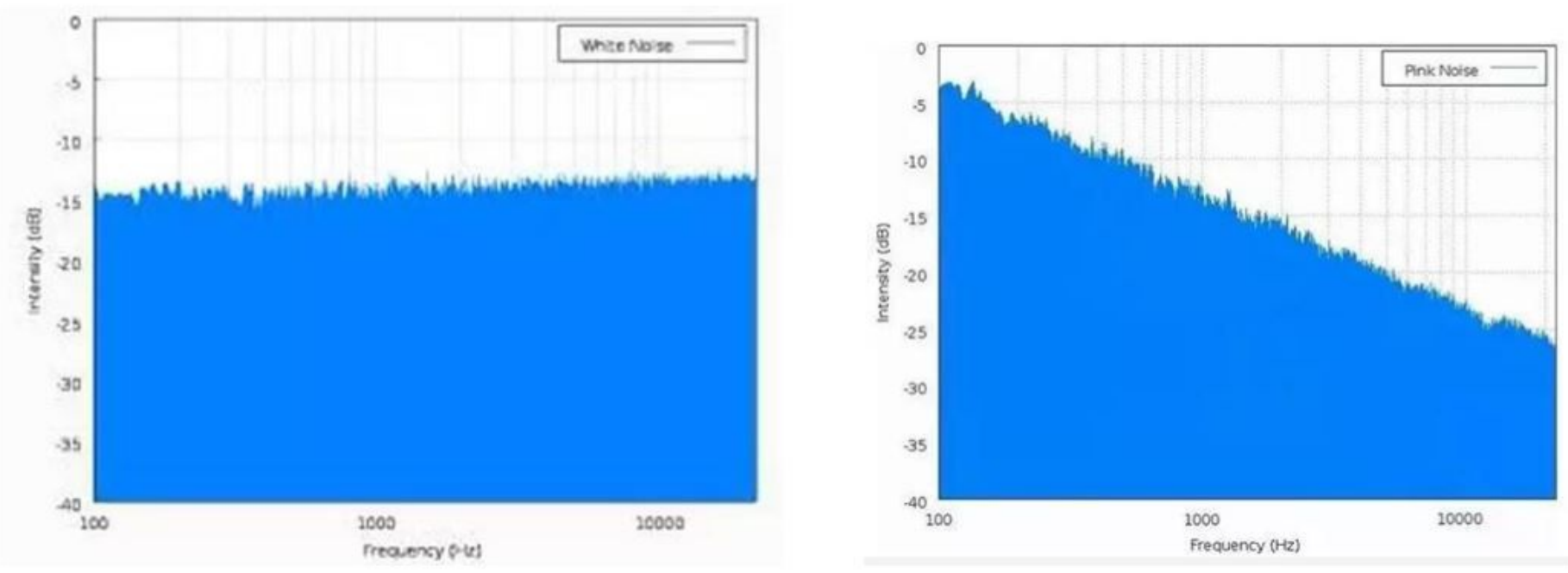

\section{Figure 1}

Spectrum comparison of white noise and pink noise 


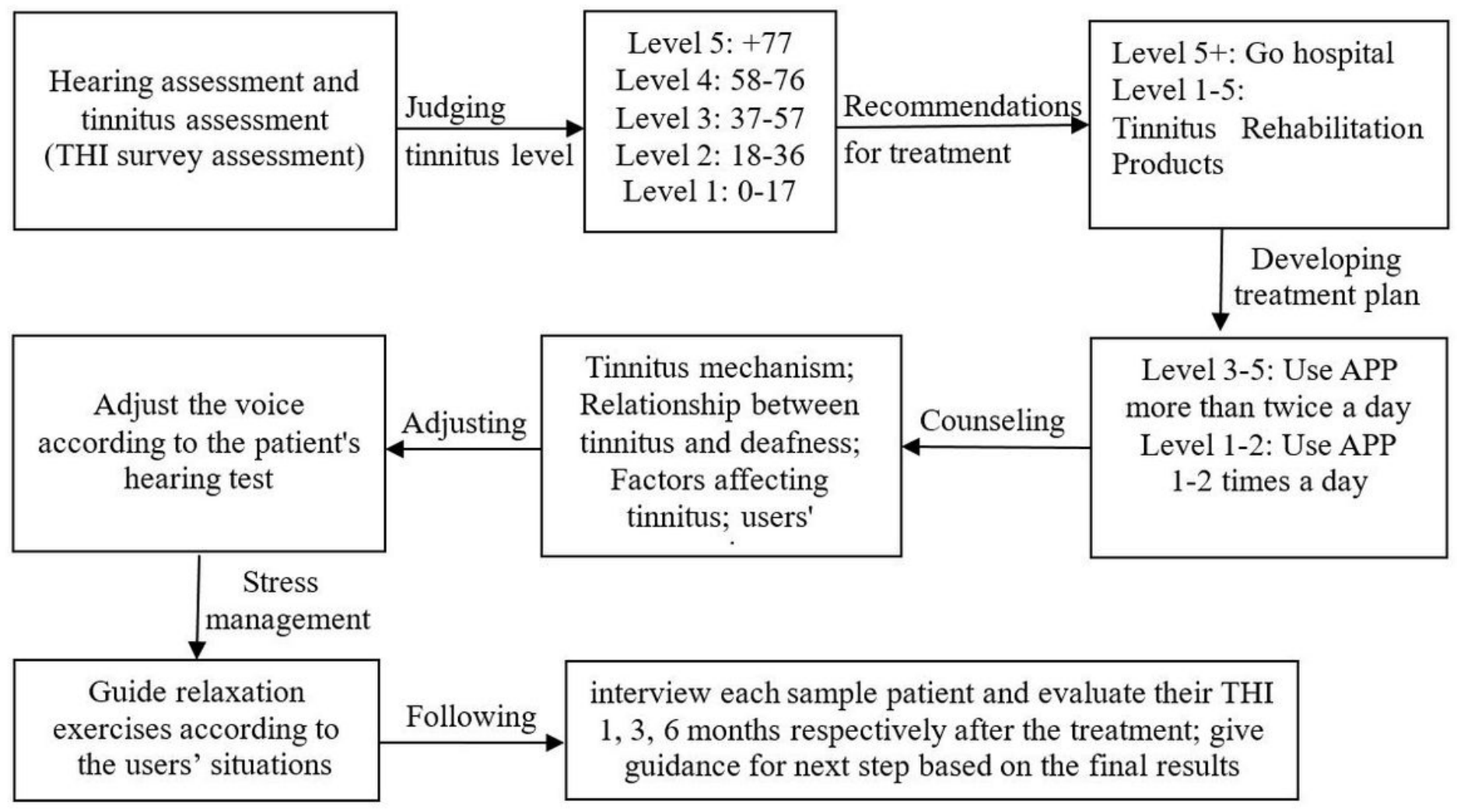

Figure 2

Flow chart of tinnitus rehabilitation treatment

\section{Supplementary Files}

This is a list of supplementary files associated with this preprint. Click to download.

- S1Researchreportform.docx

- S2EthicalapprovalforclinicalresearchofFractalMusicTherapy.pdf

- S3Informedconsentofsubjects.docx

- S4Acceptance20X110.pdf

- S5ApplicationBTE.pdf

- S6Data.xlsx

- S7Table.docx

- S8Pictures.docx 